\title{
Schlafbezogene Atmungsstörungen - Neuauflage der Internationalen Klassifikation von Schlafstörungen (ICSD-2) der American Academy of Sleep Medicine (AASM)
}

\author{
Sleep-Related Breathing Disorders - Second Edition of the International Classification \\ of Sleep Disorders (ICSD-2) of the American Academy of Sleep Medicine (AASM)
}

Autor

Institut

\section{H.-W. Duchna}

Berufsgenossenschaftliche Kliniken Bergmannsheil, Klinikum der Ruhr-Universität-Bochum, Medizinische Klinik III, Bochum eingereicht 3.1.2006

akzeptiert 15.2. 2006

Bibliografie

DOI $10.1055 / \mathrm{s}-2006-944248$

Pneumologie 2006; 60; 568-575

(c) Georg Thieme Verlag KG

Stuttgart · New York

ISSN 0934-8387

Korrespondenzadresse

Priv.-Doz. Dr. med.

Hans-Werner Duchna

Berufsgenossenschaftliche Kliniken Bergmannsheil,

Klinikum der Ruhr-Universität-

Bochum · Medizinische Klinik III, Pneumologie, Allergologie,

Schlaf- und Beatmungsmedizin Bürkle-de-la-Camp-Platz 1 44789 Bochum

hans-werner.duchna@rub.de

\section{Zusammenfassung}

Im Jahr 2005 erfolgte eine Neuauflage der 1990 publizierten Internationalen Klassifikation von Schlafstörungen (ICSD) durch die American Academy of Sleep Medicine (AASM). Es ist anzunehmen, dass diese zweite Version der ICSD, die ICSD-2, zukünftig auch in Deutschland gelten wird. Die ICSD-2 hat das Ziel, die derzeit bekannten Schlaf- und Aufwachstörungen wissenschaftlich fundiert und evidenzbasiert zu beschreiben und zu klassifizieren. Gleichzeitig sollte die ICSD-2 kompatibel zu international gebräuchlichen Systemen der Klassifikation von Krankheiten sein (ICD-9, ICD-10). Dieser Artikel stellt eine zusammenfassende und komprimierende Übersetzung der in der revidierten Internationalen Klassifikation von Schlafstörungen der Amerikanischen Schlafgesellschaft (AASM) aufgeführten schlafbezogenen Atmungsstörungen dar.

\section{Einleitung - Unterschiede zwischen ICSD-1 und ICSD-2}

Aufgrund der großen Heterogenität der Schlafstörungen und des unterschiedlichen Wissenstandes bezüglich unterschiedlicher Schlafstörungen wurde das axiale System der ersten Version der ICSD zugunsten einer pragmatischen $\mathrm{Zu}$ ordnung der Schlaf- und Aufwachstörungen in acht Kategorien geändert ( Tab. 1). Prinzipiell unterscheidet sich die ICSD-2 in folgenden Punkten vom alten System (ICSD-1 [1]):

Die ICSD-2 ist kein axial aufgebautes System.

Aufgrund der großen Variabilität in den einzelnen Schlafzentren enthält die ICSD-2 keine Auflistung der Vorgehensweise zur Diagnosestellung einer Schlafstörung.

Die Begriffe „intrinsische Dyssomnien“ und „extrinsische Dyssomnien“ wurden zuguns-

\section{Abstract}

In 2005 the American Academy of Sleep Medicine (AASM) published a revised form of the International Classification of Sleep Disorders (ICSD-2). Goals of the ICSD-2 are: A) To describe all currently recognized sleep and arousal disorders, and to base the descriptions on scientific and clinical evidence. B) To present the sleep and arousal disorders in an overall structure that is rational and scientifically valid. C) To render the sleep and arousal disorders as compatible with ICD-9 and ICD-10 as possible. In this article, sleep-disordered breathing disorders, as classified in the ICSD-2 are presented. ten der Einteilung der Schlafstörung in acht Kategorien aufgegeben.

Die sekundären Schlafstörungen, das heißt diejenigen, die auf internistische, neurologische, psychiatrische oder andere Erkrankungen zurückzuführen sind, sind in ICSD-2 nicht mehr vollständig aufgeführt, da die Schlafstörung häufig nur ein Symptom der Grunderkrankung darstellt.

In der ICSD-2 werden definitive Diagnosekriterien aufgeführt. Eine entsprechende Schlafstörung sollte nur bei Erfüllung aller Kriterien diagnostiziert werden. Eine Schweregradeinteilung wurde aufgrund des Fehlens einheitlicher Merkmale nicht vorgenommen.

Die Präsentation der einzelnen Schlafstörung erfolgt weitgehend einheitlich, unterteilt in folgende Unterkapitel: alternative Bezeichnungen, essenzielle Symptome, assoziierte Symptome, Epidemiologie, prädisponierende Faktoren, Genetik, 
Tab. 1 Einteilung der Schlafstörungen in acht Kategorien gemäß ICSD-2 [1]

\section{Schlafstörungen gemäß ICSD-2}

I. Insomnien

II. schlafbezogene Atmungsstörungen

III. Hypersomnien zentralen Ursprungs, nicht bedingt durch Störungen der zirkadianen Rhythmik, schlafbezogene Atmungsstörungen oder andere Gründe für einen gestörten Nachtschlaf

IV. Störungen der zirkadianen Rhythmik

V. Parasomnien

VI. schlafbezogene Bewegungsstörungen

VII. einzelne Symptome, Normvarianten und ungelöste Fragestellungen

VIII. andere Schlafstörungen

Verlauf und Komplikationen, Pathologie und Pathophysiologie, Polysomnographie und andere objektive Parameter, Diagnosekriterien, Subtypen, ungelöste Fragen und zukünftige Entwicklung, Differenzialdiagnose und Literaturangaben.

\section{Schlafbezogene Atmungsstörungen}

In der Kategorie II der ICSD-2 werden schlafbezogene Störungen der Respiration einer von 14 Krankheiten zugeordnet ( Tab. 2). Diagnostischer Goldstandard ist neben der ausführlichen Anamnese die Polysomnographie, ggf. ergänzt durch weiterführende internistische, neurologische, pädiatrische, Hals-Nasen-Ohrenärztliche, Mund-Kiefer-Gesichts-chirurgische sowie neuropsychologische und neurophysiologische Untersuchungen. Die Einteilung der schlafbezogenen Atmungsstörungen erfolgt in erster Linie aufgrund der Ätiologie und Pathophysiologie, wobei pharyngeale Obstruktionen, verschiedene Formen der pulmonalen Gasaustauschstörung, zentrale und periphere Störungen der At-

Tab. 2 Kategorie II: Schlafbezogene Atmungsstörungen gemäß ICSD-2 [1]

\section{Schlafbezogene Atmungsstörungen (ICSD-2)}

\section{Zentrale Schlafapnoe-Syndrome}

1. primäre zentrale Schlafapnoe

2. zentrale Schlafapnoe mit Cheyne Stokes Atmungsmuster

3. zentrale Schlafapnoe bei Höhenaufenthalt

4. zentrale Schlafapnoe bei internistischen/neurologischen Erkrankungen (außer Cheyne Stokes-Atmung)

5. zentrale Schlafapnoe bei Drogen- oder Medikamentengebrauch

6. primäre Schlafapnoe in der frühen Kindheit

\section{Obstruktive Schlafapnoe-Syndrome}

7. obstruktive Schlafapnoe, Erwachsene

8. obstruktive Schlafapnoe, Pädiatrie

Schlafbezogene Hypoventilation/hypoxämische Syndrome

9. schlafbezogene nicht-obstruktive alveoläre Hypoventilation, idiopathisch

10. kongenitales zentrales alveoläres Hypoventilations-Syndrom Schlafbezogene Hypoventilation/Hypoxämie bei internistischen/ neurologischen Erkrankungen

11. schlafbezogene Hypoventilation/Hypoxämie bei Lungenparenchym- oder Gefäß-Erkrankungen

12. schlafbezogene Hypoventilation/Hypoxämie bei obstruktiver Atemwegserkrankung

13. schlafbezogene Hypoventilation/Hypoxämie bei neuromuskulären Erkrankungen oder Thoraxerkrankungen

Andere schlafbezogene Atmungsstörungen

14. Schlafapnoe/schlafbezogene Atmungsstörung, unklassifiziert mungspumpe Berücksichtigung finden. Wie bisher wird zwischen zentralen Apnoen, definiert durch Atemstillstände bei ausbleibender Atemanstrengung, und obstruktiven Apnoen, gekennzeichnet durch fortgeführte Atemanstrengung bei Sistieren der Ventilation, unterschieden. Eine alveoläre Hypoventilation wird bei einem Kohlendioxid-Partialdruck $\left(\mathrm{PaCO}_{2}\right)$ oberhalb $45 \mathrm{~mm} \mathrm{Hg}$ angenommen. Da dieser Grenzwert anhand von arteriellen Messungen im Wachzustand definiert wurde, ist eine klare Abgrenzung des physiologischen Anstiegs des $\mathrm{PaCO}_{2}$ während des Schlafs $(6-8 \mathrm{~mm} \mathrm{Hg})$ von der pathologischen nächtlichen Hypoventilation in der Literatur nicht exakt definiert. Daher wird der Begriff der schlafbezogenen nächtlichen Hypoventilation gefordert um klarzustellen, dass hiermit nicht der im Wachzustand gemessene Grad der Hypoventilation gemeint ist. Die alveoläre Hypoventilation wird von der AASM als chronisch bezeichnet, wenn eine nächtliche Hyperkapnie über einen Zeitraum von mindestens 1 Monat besteht.

\section{Zentrale Schlafapnoe-Syndrome}

Zu dieser Gruppe werden die in $\bullet$ Tab. 2 aufgeführten 6 Krankheitsbilder gezählt. Charakteristisch für die primäre zentrale Schlafapnoe, die gehäuft bei Älteren diagnostiziert wird, sind polysomnographisch gesicherte wiederholte nächtliche zentrale Apnoen ( $>10$ Sekunden) mit einem Apnoe-Index (AI) $\geq 5$ zentrale Apnoen/Stunde. Die Ursache hierfür ist unklar (idiopathisch), die Patienten weisen am Tage typischerweise einen $\mathrm{PaCO}_{2}$ unter $40 \mathrm{~mm} \mathrm{Hg}$ und eine gesteigerte Atemantwort auf $\mathrm{CO}_{2}$ auf. Allen zentralen Schlafapnoe-Syndromen gemeinsam ist eine Instabilität der Atmungskontrolle, die zu einem Abatmen von $\mathrm{CO}_{2}$ unter die Apnoeschwelle mit konsekutiver Entstehung zentraler Apnoen führt. Die Apnoe wird durch eine Weckreaktion (Arousal) nach entsprechendem Anstieg des $\mathrm{PaCO}_{2}$ bzw. Abfall des $\mathrm{PaO}_{2}$ beendet. Eine Insomnie, verbunden mit gehäuften Schlaf-WachÜbergängen in der Nacht und konsekutiv gesteigerter Instabilität der Atmungskontrolle wird ebenso wie die nasale Obstruktion als Verstärkungsfaktor für das Auftreten zentraler Apnoen diskutiert. Neben der durch die Schlaffragmentierung bedingten Tagesmüdigkeit ist wenig über Epidemiologie und den Verlauf dieser Erkrankung sowie mögliche Auswirkungen auf das kardiovaskuläre System bekannt. Die von der AASM minimal geforderten Diagnosekriterien sind in $\bullet$ Tab. 3 aufgeführt [2 - 7]. Im Gegensatz zur primären zentralen Schlafapnoe, bei der abrupte Apnoe- und kurze Hyperpnoephasen auftreten, weisen Patienten mit zentraler Schlafapnoe mit Cheyne-Stokes-Atmungsmuster ein charakteristisches Crescendo-Decrescendo-Muster ihrer Atmung mit verlängerten Hyperpnoephasen auf. Typischerweise tritt die Cheyne-Stokes-Atmung im non-REM (rapid-

Tab.3 Diagnosekriterien für die primäre zentrale Schlafapnoe (ICSD-2 [1])

Diagnosekriterien: Primäre zentrale Schlafapnoe (ICSD-2)

Anamnese (mindestens eines der folgenden Kriterien):

exzessive Tagesmüdigkeit

nächtliche Weckreaktionen/Erwachen oder Beschwerden einer Insomnie

nächtliches Erwachen mit Atemnot

Polysomnographie: $\geq 5$ zentrale Apnoen/h

Erkrankung nicht besser beschrieben durch andere Schlafstörung, eine internistische oder neurologische Erkrankung, Medikamenten-/ Drogengebrauch 
Tab. 4 Diagnosekriterien für die zentrale Schlafapnoe mit Cheyne-StokesAtmung (ICSD-2 [1])

\section{Diagnosekriterien: Zentrale Schlafapnoe mit Cheyne Stokes Atmungsmuster (ICSD-2)}

Polysomnographie: $\geq 10$ zentrale Apnoen/h mit Crescendo-Decrescendo Muster der Atmung assoziiert mit gehäuften Weckreaktionen und gestörter Schlafstruktur

fakultativ: exzessive Tagesmüdigkeit, insomnische Beschwerden, nächtliches Erwachen mit Atemnot

Assoziation mit schwerer internistischer/neurologischer Erkrankung (Herzinsuffizienz, Niereninsuffizienz, Apoplex)

Erkrankung nicht besser beschrieben durch andere Schlafstörung oder Medikamenten-/Drogengebrauch

eye-movement) Schlaf auf und kann zu exzessiver Tagesmüdigkeit, insomnischen Beschwerden oder nächtlichen Atemnotbeschwerden führen ( Tab.4). Die Erkrankung steht in ursächlichem Zusammenhang mit unterschiedlichen Formen der Herzinsuffizienz, Apoplex oder einem Nierenversagen. Als Risikofaktoren gelten das männliche Geschlecht, ein Alter > 60 Jahre, Vorhofflimmern und Hypokapnie (Tages- $\mathrm{PaCO}_{2} \leq 38 \mathrm{~mm} \mathrm{Hg}$ ). Neben den oben aufgeführten pathophysiologischen Erwägungen ist eine initiale Hyperventilation, ausgelöst durch eine Reizung vagaler Lungendehnungsrezeptoren bei Lungenstauung (Herzinsuffizienz/Nierenversagen?) oder zentraler Genese (Apoplex?) für die Entstehung der zentralen Schlafapnoe mit Cheyne-Stokes-Atmung bedeutsam. Hinzu kommt eine verlängerte LungenChemorezeptor-Kreislaufzeit, direkt proportional zur Länge des Apnoe-Hyperventilations-Zyklus (im Allgemeinen > 45 Sekunden), die den graduellen Auf- und Abbau des Atemantriebs erklärt. Während die Cheyne-Stokes-Atmung bei herzinsuffizienten Patienten einen negativen prognostischen Wert (Transplantationsrate, Tod) hat, ist die klinische Bedeutung, aber auch die Pathophysiologie bei Apoplex und Nierenversagen weitgehend ungeklärt [8-13].

Essentielles Kennzeichen der zentralen Schlafapnoe bei Höhenaufenthalt ist der klare Bezug zu einem Aufstieg in Höhen oberhalb von ca. 4000 Metern. Die Erkrankung tritt üblicherweise während der ersten Nacht in entsprechender Höhe auf und spiegelt die gesteigerte Atemantwort bei Hypoxie wider, wobei die Geschwindigkeit des Aufstiegs, die erreichte Höhe und eine individuelle Prädisposition modulatorisch wirken (Tab.5 [14-19]).

In der Rubrik zentrale Schlafapnoe bei internistischen/neurologischen Erkrankungen werden vorwiegend Erkrankungen mit Stammhirnläsionen vaskulären, degenerativen, demyelinisierenden oder traumatischen Ursprungs subsummiert, die eine zentrale Apnoe ohne Cheyne-Stokes-Atmung bedingen. Störun-

Tab. 5 Diagnosekriterien für die zentrale Schlafapnoe bei Höhenaufenthalt (ICSD-2 [1])

\section{Diagnosekriterien: Zentrale Schlafapnoe bei Höhenaufenthalt (ICSD-2)}

Kürzlicher Aufstieg in Höhen oberhalb von 4000 Metern Polysomnographie: $\geq 5$ zentrale Apnoen/h im NREM-Schlaf Zykluslänge: $12-34 \mathrm{~s}$
Tab. 6 Diagnosekriterien für die zentrale Schlafapnoe bei Drogen- oder Medikamentengebrauch (ICSD-2 [1])

\section{Diagnosekriterien: Zentrale Schlafapnoe bei Drogen- oder Medikamentengebrauch (ICSD-2)}

Regelmäßige Einnahme eines langwirksamen Opioids $\geq 2$ Monate Polysomnographie: $\geq 5$ zentrale Apnoen/h oder $\geq 10$ zentrale Apnoen oder Hypopnoen/h mit Crescendo-Decrescendo Muster der Atmung assoziiert mit gehäuften Weckreaktionen und gestörter Schlafstruktur

Erkrankung nicht besser beschrieben durch andere Schlafstörung oder eine internistische/neurologische Erkrankung

Tab. 7 Diagnosekriterien für die primäre Schlafapnoe in der frühen Kindheit (ICSD-2 [1])

\section{Diagnosekriterien: Primäre Schlafapnoe in der frühen Kindheit} (ICSD-2)

Apnoe bei Frühgeborenen: Prolongierte zentrale Apnoen ( $\geq 20 \mathrm{~s}$ ) oder kürzere Apnoen (einschließlich gemischter und obstruktiver Apnoen) mit signifikantem Abfall der Herzfrequenz, Hypoxämie, klinischen Symptomen oder Interventionsnotwendigkeit; Kind < 37 Wochen Konzeptionsalter

Apnoe in der frühen Kindheit: Prolongierte zentrale Apnoen ( $\geq 20 \mathrm{~s}$ ) oder kürzere Apnoen (einschließlich gemischter und obstruktiver Apnoen) mit Bradykardie, Zyanose, Blässe oder ausgeprägter Hypotonie; Kind $\geq 37$ Wochen Konzeptionsalter

Erkrankung nicht besser beschrieben durch andere Schlafstörung, eine internistische/neurologische Erkrankung oder Medikamente

gen der kardialen oder renalen Funktion können ebenfalls in dieser Gruppe aufgeführt werden. Unter einer zentralen Schlafapnoe bei Drogen- oder Medikamentengebrauch werden in erster Linie durch Morphium oder dessen Derivate induzierte nächtliche Atmungsstörungen verstanden. Die diagnostischen Kriterien werden in Tab. 6 aufgeführt [20-22].

Gesondert betrachtet wird die primäre Schlafapnoe in der frühen Kindheit ( Tab. 7). Die primäre Schlafapnoe in der frühen Kindheit umfasst zentrale, gemischte und auch obstruktive Apnoenphasen oder Hypopnoen. Insbesondere bei Frühgeborenen ist diese Erkrankung gehäuft anzutreffen (Prävalenz ca. 25\% bei einem Geburtsgewicht < 2500 Gramm; ca. $84 \%$ bei einem Geburtsgewicht $<1000 \mathrm{Gramm}$ ) und auf eine Unreife des Atmungszentrums im Stammhirn zurückzuführen (daher auch unter „Zentrale Schlafapnoe-Syndrome“ aufgelistet). Das Spektrum dieser Erkrankung umfasst jedoch auch den Zeitraum nach der Neugeborenenphase (bis 4 Wochen nach der Geburt) zeitgerecht geborener Kinder. Beim Neugeborenen bzw. Kleinkind kann die Schlafapnoe durch unterschiedliche Faktoren, wie Anämie, Infekte, Hypoxämie, metabolische Störungen, gastroösophagealer Reflux, Medikamente oder eine Narkose ausgelöst werden. Ein Zusammenhang mit dem plötzlichen Kindstod wird gesehen (near-miss sudden infant death syndrome [near-SIDS]; apparent life-threatening event [ALTE]), die primäre Schlafapnoe in der frühen Kindheit ist jedoch klar von den Begriffen ALTE/SIDS abzugrenzen und ist auch nicht als unabhängiger Risikofaktor des plötzlichen Kindstodes bewiesen. Trotz der Heterogenität der zugrunde liegenden Pathophysiologie wird in den meisten Studien eine progressive Abnahme der Krankheitsprävalenz mit zunehmendem Alter der Kinder beschrieben [23 - 29]. 


\section{Obstruktive Schlafapnoe-Syndrome}

Charakteristisch für die obstruktive Schlafapnoe sind rezidivierende komplette (Apnoe) oder inkomplette (Hypopnoe) Obstruktionen der oberen (extrathorakalen) Atemwege im Schlaf. Definitionsgemäß dauert eine Apnoe/Hypopnoe mindestens 10 Sekunden, kann jedoch auch eine Minute und länger anhalten. Üblicherweise führen die repetitiven Apnoe- bzw. Hypopnoephasen zu einem Abfall der Sauerstoffsättigung im Blut; beendet werden sie jeweils durch eine kurze Weckreaktion (Arousal). Leitsymptome des Erwachsenen sind nächtliches Schnarchen, beobachtete Atemaussetzer und eine exzessive Tagesmüdigkeit mit konsekutiv eingeschränkter Lebensqualität unabhängig von der Schlafdauer. Häufig von den Bettpartnern und den Betroffenen berichtet und durch Studien belegt wird eine Symptomsteigerung nach Gewichtszunahme und Alkoholgenuss vor dem Schlaf. Das obstruktive Schlafapnoe-Syndrom (OSAS) zeigt aufgrund eines erhöhten Sympathikotonus sowohl in der Nacht als auch am Tage und proatherogener Effekte auf die Blutgefäße eine starke Assoziation zu kardiovaskulären Erkrankungen. Die Evidenz für das OSAS als unabhängiger Risikofaktor für die systemische arterielle Hypertonie ist aufgrund der aktuellen Datenlage gut belegt. Kausale Zusammenhänge zwischen OSAS und Herzrhythmusstörungen, koronarer Herzerkrankung, Apoplex, pulmonaler Hypertonie und metabolischen Erkrankungen (z.B. Diabetes mellitus) sind beschrieben. Bei Patienten mit dilatativer Kardiomyopathie oder ischämisch bedingter Herzinsuffizienz führt ein koprävalentes OSAS häufig zu einer Verschlechterung der kardialen Erkrankung mit Entwicklung einer kongestiven Herzinsuffizienz. Das OSAS tritt in allen Altersgruppen, bevorzugt jedoch im mittleren und höheren Lebensalter, auf. In der Gruppe der 30- bis 60-Jährigen wird die Prävalenz des OSAS auf 4 Prozent der Männer und 2 Prozent der Frauen geschätzt. Die dem OSAS zugrunde liegende Pathophysiologie der Einengung der extrathorakalen oberen Atemwege ist multifaktoriell. So sind die oberen Atemwege bei Patienten mit einem OSAS neben entsprechenden knöchernen kraniofazialen anatomischen Verhältnissen häufig auf der Ebene des weichen Gaumens, der Zunge und der lateralen Pharynxwände durch Weichteilgewebe eingeengt. Ursächlich hierfür ist in erster Linie ein erhöhter Body-Mass-Index mit Fetteinlagerung, gelegentlich auch vergröBerte Rachenmandeln, ein Down-Syndrom, Akromegalie oder Hypothyreose sowie inhalativer Nikotingebrauch (chronische Pharyngitis). Im Schlaf führt die physiologische Erschlaffung dilatierend wirkender Pharynxmuskeln zusätzlich zu einer weiteren Lumenverengung des Pharynx mit konsekutiver Entstehung der Apnoen und Hypopnoen. In einigen Fällen führt die pharyngeale Lumeneinengung lediglich zu Schnarchen mit erhöhter Atemarbeit (polysomnographisch dokumentiert durch Ösophagusdruckmessung) ohne das Vollbild eines OSAS mit Apnoen und Hypopnoen. Da dieses obstruktive Schnarchen (UARS: Upper Airway Resistance Syndrome) ebenfalls mit nächtlichen Weckreaktionen (RERA: Respiratory Effort Related Arousal), gestörter Schlafstruktur und exzessiver Tagesmüdigkeit einhergeht, wird es ebenfalls dem obstruktiven Schlafapnoe-Syndrom zugeordnet ( Tab. $8[30-35])$.

Bei Kindern manifestiert sich die obstruktive Schlafapnoe häufig in Form einer prolongierten partiellen Obstruktion der oberen Atemwege (vergleichbar UARS beim Erwachsenen) oder intermittierend auftretender Schlafphasen mit obstruktiven Apnoen oder Hypopnoen. Damit assoziiert werden Sauerstoffdesaturationen, Hyperkapnie und Weckreaktionen, die anders als beim
Tab. 8 Diagnosekriterien für die obstruktive Schlafapnoe bei Erwachsenen (ICSD-2 [1])

\section{Diagnosekriterien: Obstruktive Schlafapnoe, Erwachsene (ICSD-2). Gefordert sind: A + B + D oder C + D}

Anamnese (mindestens eines der folgenden Kriterien):

1. ungewollte Einschlafepisoden während Wachheit, Tagesmüdigkeit, nicht-erholsamer Schlaf oder Insomnie

2. nächtliche Erwachen mit Atemstillstand, Erstickungsanfällen, nach Luft schnappen

3. durch Bettpartner beobachtetes lautes Schnarchen oder Atemstillstände im Schlaf

Polysomnographie: $\geq 5$ respiratorische Ereignisse (Apnoen, Hypopnoen, RERAs/h mit Atemanstrengung bei jedem respiratorischen Ereignis

oder

Polysomnographie: $\geq 15$ respiratorische Ereignisse (Apnoen, Hypopnoen, RERAs/h mit Atemanstrengung bei jedem respiratorischen Ereignis

Erkrankung nicht besser beschrieben durch andere Schlafstörung, eine internistische oder neurologische Erkrankung, Medikamenten-/ Drogengebrauch

Erwachsenen vornehmlich durch Bewegungen und Veränderungen autonomer Funktionen (z. B. Herzfrequenz) auffallen, beobachtet. Hauptsächlich werden von den Eltern nächtliches Schnarchen und eine erschwerte Atmung während des Schlafs beschrieben. Aufgrund des weichen Brustkorbes wird häufig eine paradoxe Atmung mit inspiratorischer Einziehung des Sternums beobachtet ( Tab.9). Die Prävalenz der obstruktiven

Tab.9 Diagnosekriterien für die obstruktive Schlafapnoe bei Kindern (ICSD-2 [1])

\section{Diagnosekriterien: Obstruktive Schlafapnoe, Pädiatrie (ICSD-2)}

Anamnese der Eltern: Schnarchen oder erschwerte (behinderte) Atmung im Schlaf

Anamnese der Eltern (mindestens eines der folgenden Kriterien): paradoxe Atmung mit inspiratorischer Einziehung des Sternums Weckreaktionen mit Bewegung (movement arousals)

nächtliches Schwitzen (Diaphorese)

Dorsalextension des Halses im Schlaf

exzessive Tagesmüdigkeit, Hyperaktivität oder aggressives Verhalten Wachstumsretardierung

morgendliche Kopfschmerzen

sekundäre Enuresis

Polysomnographie: $\geq 1$ respiratorisches Ereignis (Apnoe, Hypopnoe)/h mit einer Länge $\geq 2$ Atmungszyklen

Polysomnographie (entweder 1. oder 2.):

1. (mindestens eines der folgenden Kriterien): gehäufte Weckreaktionen (Arousals) assoziiert mit erhöhter Atemanstrengung

arterielle Sauerstoffdesaturation in Assoziation mit apnoeischen Episoden

Hyperkapnie im Schlaf

ausgeprägt erhöhter inspiratorischer Sog (Ösophagusdruckmessung)

2. Hyperkapnie, $\mathrm{O}_{2}$-Desaturationen oder beides im Schlaf assoziiert mit Schnarchen, paradoxer Atmung mit inspiratorischer Einziehung des Sternums + mindestens eines der folgenden Kriterien: gehäufte Weckreaktionen aus dem Schlaf (Arousals) ausgeprägt erhöhter inspiratorischer Sog (Ösophagusdruckmessung)

Erkrankung nicht besser beschrieben durch andere Schlafstörung, eine internistische oder neurologische Erkrankung, Medikamenten-/ Drogengebrauch 
Schlafapnoe wird auf 2 Prozent bei ansonsten gesunden Kindern geschätzt, mit einer Erstmanifestation der Symptome in den frühen Lebensjahren. Neben pharyngealen und kraniofazialen Anomalien, dem Down-Syndrom und Übergewichtigkeit stellen vergrößerte Gaumen- und Rachenmandeln einen wichtigen pathophysiologischen Faktor in der Entstehung der obstruktiven Schlafapnoe im Kindesalter dar. Familiäre Häufungen des OSAS sind beschrieben, die Bedeutung von Genetik und Umwelteinflüssen jedoch noch nicht geklärt. Mögliche Komplikationen der Erkrankung sind Wachstumsstörungen, kognitive und Verhaltensstörungen wie geistige Entwicklungsstörungen, schlechte Schulleistungen, Aufmerksamkeits-Defizit/Hyperaktivitäts-Syndrom oder aggressives Verhalten. Die möglichen kardiovaskulären Auswirkungen des OSAS bei Kindern entsprechen vermutlich denjenigen bei Erwachsenen, diesbezüglich beweisende, lang angelegte prospektive kontrollierende Studien stehen allerdings noch aus [36-41].

\section{Schlafbezogene Hypoventilation/hypoxämische Syndrome}

Zwei Formen der schlafbezogenen Hypoventilation/hypoxämischen Syndrome werden unterschieden: Zum einen die schlafbezogene nicht-obstruktive alveoläre Hypoventilation, die häufig idiopathisch auftritt und das kongenitale zentrale alveoläre Hypoventilations-Syndrom. Beiden Erkrankungen gemeinsam ist eine gestörte Kontrolle der Ventilation mit reduzierter Steigerung der Atemantwort auf Hyperkapnie oder Hypoxie. Die schlafbezogene nicht-obstruktive alveoläre Hypoventilation tritt häufig erstmals in der Jugend oder beim jungen Erwachsenen in Erscheinung mit Zeichen der ventilatorischen Insuffizienz (morgendliche Kopfschmerzen, Rechtsherzbelastungszeichen, periphere Ödeme, Polyzythämie) und den Folgen eines gestörten Nachtschlafs (Schlafstörungen, Tagesmüdigkeit). Die Diagnose wird nur nach Ausschluss einer pulmonalen, endokrinologischen, neurologischen bzw. neuromuskulären, kardialen oder anderen erkennbaren Störungen der Atmungspumpe (z.B. Rippenserienfraktur) gestellt ( Tab. 10). Pathophysiologisch werden Störungen der medullären Chemorezeptorfunktion sowie Affektionen des Stammhirns durch Infarzierung, Blutung oder Poliomyelitis angenommen. Die kongenitale zentrale alveoläre Hypoventilation (früher: Undine's Fluch-Syndrom) beruht wahrscheinlich auf einer fehlerhaften Integration von Chemorezeptorafferenzen im Stammhirn und tritt üblicherweise von Geburt an in Erscheinung. Das ansonsten gesund erscheinende Neugeborene zeigt keine adäquate Spontanatmung und muss intubiert und beatmet werden. In einigen Fällen manifestiert sich die Er-

Tab. 10 Diagnosekriterien für die (idiopathische) schlafbezogene nicht-obstruktive alveoläre Hypoventilation, (ICSD-2 [1])

\section{Diagnosekriterien: Schlafbezogene nicht-obstruktive alveoläre Hypoventilation, idiopathisch (ICSD-2)}

Polysomnographie: Hypoventilationsphasen $>10 \mathrm{~s}$ im Schlaf assoziiert mit $\mathrm{O}_{2}$-Desaturation und gehäuften Weckreaktionen (Arousals) Ausschluss einer Ventilationsstörung bedingt durch primäre Lungenkrankheit, Skelettmalformität, neuromuskuläre Erkrankung Erkrankung nicht besser beschrieben durch andere Schlafstörung, eine internistische oder neurologische Erkrankung, Medikamenten-/ Drogengebrauch
Tab.11 Diagnosekriterien für das kongenitale zentrale alveoläre Hypoventilations-Syndrom (ICSD-2)

\section{Diagnosekriterien: Kongenitales zentrales alveoläres Hypoventilations-Syndrom (ICSD-2)}

Von Geburt an oberflächliche Atmung (Hypoventilation), oder Zyanose und Apnoen im Schlaf

Hypoventilation im Schlaf ausgeprägter als im Wachzustand Abgeschwächte oder nicht vorhandene Atemantwort auf Hypoxie oder Hyperkapnie

Polysomnographie: schwere Hyperkapnie und Hypoxie, vornehmlich ohne Apnoen

Erkrankung nicht besser beschrieben durch andere Schlafstörung, eine internistische oder neurologische Erkrankung, Medikamenten-/ Drogengebrauch

Tab. 12 Diagnosekriterien für schlafbezogene Hypoventilation/Hypoxämie bei Lungenparenchym- oder Gefäßerkrankungen (ICSD-2 [1])

Diagnosekriterien: Schlafbezogene Hypoventilation/Hypoxämie bei Lungenparenchym- oder Gefäßerkrankungen (ICSD-2)

Eine Lungenparenchym- oder Gefäßerkrankung ist die primäre Ursache der nächtlichen Hypoxämie

Polysomnographie oder Blutgasanalyse im Schlaf (mindestens ein Kriterium):

1. $\mathrm{O}_{2}$-Sättigung $\left(\mathrm{SaO}_{2}\right)$ im Schlaf $<90 \%>5$ min. mit einem Nadir $\leq 85 \%$

2. $\mathrm{SaO}_{2}$ im Schlaf $<90 \%$ während $>30 \%$ der Gesamtschlafzeit

3. Blutgasanalyse im Schlaf zeigt $\mathrm{PaCO}_{2}$-Wert, der abnormal hoch oder dysproportional zum Wach- $\mathrm{PaCO}_{2}$ angestiegen ist

Erkrankung nicht besser beschrieben durch andere Schlafstörung, eine internistische oder neurologische Erkrankung, Medikamenten-/ Drogengebrauch

krankung in Form von Hypoventilationsphasen mit Hypoxämie und Hyperkapnie, wobei die Hypoventilationsphasen in der Nacht ausgeprägter als am Tage sind (๑ Tab. 11 [42 - 49]).

\section{Schlafbezogene Hypoventilation/Hypoxämie bei internistischen|neurologischen Erkrankungen}

Hierunter fallen sowohl Störungen des Gasaustausches (Ventilation, Perfusion oder Diffusion) als auch periphere Atmungspumpenstörungen bei entsprechender internistischer/neurologischer Grundkrankheit. Unterschieden werden 3 Krankheitsentitäten, die schlafbezogene Hypoventilation/Hypoxämie bei Lungenparenchym- oder Gefäßerkrankungen ( Tab.12), bei obstruktiver Atemwegserkrankung, hier wird auch das Lungenemphysem subsummiert, ( Tab.13) sowie bei neuromuskulären Erkrankungen oder Thoraxerkrankungen, hier ist auch das Obesitas-Hypoventilations-Syndrom aufgeführt ( Tab. 14). Neben den bereits eingangs aufgeführten definitorischen Schwierigkeiten einer schlafbezogenen Hypoventilation werden langanhaltende (phasische) Sauerstoffentsättigungen (ohne sägezahnartige Fluktuation) bei entsprechender Grundkrankheit in der Abwesenheit von obstruktiven, gemischten oder zentralen Apnoen oder Hypopnoen bzw. ohne inspiratorische Atemflussbehinderung (Obstruktion der extrathorakalen Atemwege) oder Schnarchen als diagnostischer Goldstandard angesehen. Werden zusätzliche obstruktive oder zentrale Apnoen diagnostiziert, so ist ein ggf. koprävalentes Schlafapnoe-Syndrom nach den oben auf- 
Tab. 13 Diagnosekriterien für schlafbezogene Hypoventilation/Hypoxämie bei obstruktiver Atemwegserkrankung (ICSD-2 [1])

\section{Diagnosekriterien: Schlafbezogene Hypoventilation/Hypoxämie bei obstruktiver Atemwegserkrankung (ICSD-2) \\ Eine obstruktive Atemwegserkrankung (einschl. Lungenemphysem) ist die primäre Ursache der nächtlichen Hypoxämie \\ Polysomnographie oder Blutgasanalyse im Schlaf (mindestens ein Kriterium): \\ 1. $\mathrm{O}_{2}$-Sättigung $\left(\mathrm{SaO}_{2}\right)$ im Schlaf $<90 \%>5$ min. mit einem Nadir $\leq 85 \%$ 2. $\mathrm{SaO}_{2}$ im Schlaf $<90 \%$ während $>30 \%$ der Gesamtschlafzeit \\ 3. Blutgasanalyse im Schlaf zeigt $\mathrm{PaCO}_{2}$-Wert, der abnormal hoch oder dysproportional zum Wach- $\mathrm{PaCO}_{2}$ angestiegen ist Erkrankung nicht besser beschrieben durch andere Schlafstörung, eine internistische oder neurologische Erkrankung, Medikamenten-I Drogengebrauch}

Tab. 14 Diagnosekriterien für schlafbezogene Hypoventilation/Hypoxämie bei neuromuskulären oder Thoraxerkrankungen (auch: Obesitas-Hypoventilations-Syndrom) (ICSD-2 [1])

Diagnosekriterien: Schlafbezogene Hypoventilation/Hypoxämie
bei neuromuskulären Erkrankungen oder Thoraxerkrankungen
(ICSD-2)
Eine neuromuskuläre Erkrankung oder eine Erkrankung des Thorax ist
die primäre Ursache der nächtlichen Hypoxämie
Polysomnographie oder Blutgasanalyse im Schlaf (mindestens ein
Kriterium):
1. $\mathrm{O}_{2}$-Sättigung $\left(\mathrm{SaO}_{2}\right)$ im Schlaf $<90 \%>5$ min. mit einem Nadir $\leq 85 \%$
2. SaO $\mathrm{S}_{2}$ im Schlaf $<90 \%$ während $>30 \%$ der Gesamtschlafzeit
3. Blutgasanalyse im Schlaf zeigt $\mathrm{PaCO}$-Wert, der abnormal hoch
oder dysproportional zum Wach-PaCO $\mathrm{CO}_{2}$ angestiegen ist
Erkrankung nicht besser beschrieben durch andere Schlafstörung,
eine internistische oder neurologische Erkrankung, Medikamenten- $/$
Drogengebrauch

geführten Kriterien ( Tab.3-9) zusätzlich anzugeben. Der Schlaf-PaCO $\mathrm{Cann}_{2}$, muss aber nicht zwingend über $45 \mathrm{~mm} \mathrm{Hg}$ liegen, in jedem Fall wird jedoch ein deutlicher Anstieg gegenüber den Wach- $\mathrm{PaCO}_{2}$-Werten gemessen. Obwohl Patienten mit manifester Gasaustauschstörung bzw. ventilatorischer Insuffizienz am Tage diese in verstärktem Ausmaß auch während des Schlafs exprimieren werden, gibt es keinen am Tage (im Wachzustand) messbaren, zuverlässigen prädiktiven Parameter für die Vorhersage einer Hypoventilation/Hypoxämie im Schlaf. Weitgehend unklar und nicht das Thema dieser Klassifikation (ICSD-2) ist auch die prognostische Bedeutung der schlafbezogenen Hypoventilation/Hypoxämie für den Krankheitsverlauf (Entwicklung von pulmonaler Hypertonie, Herzrhythmusstörungen, Polyzythämie, kognitiven Defiziten). Auch ist die Datenlage bezüglich der Indikation zur nächtlichen Sauerstofftherapie bzw. einer ventilatorischen Unterstützung (Beatmung) und deren Auswirkung auf die Prognose des Krankheitsverlaufes bisher unvollständig [50-67].

In der Rubrik „Andere schlafbezogene Atmungsstörungen“ (- Tab.2) werden schlafbezogene Atmungsstörungen klassifiziert, die nicht in die oben aufgeführten Kategorien passen.

\section{Schlafbezogene Atmungsstörungen gemäß ICSD-2 - Schlussfolgerungen}

Die Neuauflage der ICSD durch die AASM ist eine pragmatische Anleitung zur Klassifizierung von Schlaf- und Aufwachstörungen. Die in der ICSD-2 in der Kategorie II aufgeführten schlafbezogenen Atmungsstörungen (SBAS) bilden eine Brücke zwischen der Schlafmedizin und der pneumologisch orientierten Inneren Medizin. Berücksichtigt werden die den SBAS jeweils zugrunde liegenden pathophysiologischen Abläufe bzw. die vorherrschende Atmungsstörung unter den besonderen Bedingungen des Schlafs. Gleichzeitig wird auf definitorische Schwierigkeiten verwiesen und entsprechender Forschungsbedarf aufgezeigt, so z. B. bei der schlafbezogenen Hypoventilation und der Problematik fehlender Normwerte. Für den Kliniker wertvoll sind insbesondere die in Tabellenform aufgeführten klaren Diagnosekriterien. Hierdurch wird eine präzise und einheitliche Definition der SBAS ermöglicht, so z.B. bei der Unterscheidung der primären zentralen Schlafapnoe von der zentralen Schlafapnoe mit Cheyne-Stokes Atmungsmuster. Dennoch weist die AASM auf vorhandenen Wissenslücken und den entsprechenden Forschungsbedarf in weiten Bereichen der Schlafmedizin hin. Es wird ferner betont, dass verschiedene schlafbezogene Atmungsstörungen bei ein und demselben Patienten vorkommen können und entsprechend aufgeführt werden sollen. Für den Pneumologen ist die ICSDS-2 zu verstehen als eine Anleitung zur Definition und Diagnosestellung von SBAS bzw. anderer Schlaf- und Aufwachstörungen.

\section{Anmerkung}

Teilweise Übersetzung von: American Academy of Sleep Medicine. ICSD-2 - International classification of sleep disorders, 2nd ed.: Diagnostic and coding manual. Westchester, Illinois: American Academy of Sleep Medicine, 2005. Übersetzt und interpretiert von H.-W. Duchna, Bochum

\section{Literatur}

1 American Academy of Sleep Medicine. ICSD-2 - International classification of sleep disorders, 2nd ed.: Diagnostic and coding manual. Illinois: Westchester, American Academy of Sleep Medicine, 2005

2 Bradley T, McNicholas W, Rutherford R et al. Clinical and physiological heterogeneity of the central sleep apnea syndrome. Am Rev Respir Dis 1986; 134: 217-221

3 Guilleminault C, Robinson A. Central sleep apnea. Neurol Clin 1996; 14 : $611-628$

4 Roehrs T, Conway W, Wittig $R$ et al. Sleep complaints in patients with sleep-related respiratory disturbances. Am Rev Respir Dis 1985; 132: $520-525$

5 White D, Zwillich C, Pickett C et al. Central sleep apnea: improvement with acetazolamid therapy. Arch Intern Med 1982; 142: 1816- 1819

6 Xie A, Rutherford R, Rankin F et al. Hypocapnia and increased ventilatory responsiveness in patients with idiopathic central sleep apnea. Am J Respir Care Med 1995; 152: 1950-1955

7 Xie A, Wong B, Phillipson E et al. Interaction of hyperventilation and arousal in the pathogenesis of idiopathic central sleep apnea. Am J Respir Care Med 1994; 150: 489-495

8 Hall M, Xie A, Rutherford R et al. Cycle length of periodic breathing in patients with and without heart failure. Am J Respir Care Med 1996; 154: $376-381$

9 Lanfranchi P, Braghiroli A, Bosimini E et al. Prognostic value of nocturnal Cheyne-Stokes respiration in chronic heart failure. Circulation 1999; 99: 1435 - 1440 
10 Naughton $M$, Benard D, Tam A et al. The role of hyperventilation in the pathogenesis of central sleep apnea in patients with congestive heart failure. Am Rev Respir Dis 1993; 148: 330 - 338

11 Sin D, Fitzgerald F, Parker J et al. Risk factors for central and obstructive sleep apnea in 450 men and women with and without congestive heart failure. Am J Respir Crit Care Med 1999; 160: 1101 - 1106

12 Solin $P$, Roebuck T, Johns $D$ et al. Peripheral and central ventilatory respondes in central sleep apnea with and without congestive heart failure. Am J Respir Crit Care Med 2000; 162: 2194 - 2200

13 Xie A, Skatrud J, Puelo D et al. Apnea-hypopnea threshold for $\mathrm{CO}_{2}$ in patients with congestive heart failure. Am J Respir Crit Care Med 2002; 165: $1245-1250$

14 Anholm J, Powles A, Downey R et al. Operation Everest II: arterial oxygen saturation and sleep at extreme simulated altitude. Am Rev Respir Dis 1992; 145: 817 - 826

15 Goldberg $S$, Schoene $R$, Haynor D et al. Brain tissue $\mathrm{pH}$ and ventilatory acclimatization to high altitude. J Appl Physiol 1992; 72: 58 - 63

16 Lahiri S, Barnard P. Role of arterial chemoreflex in breathing during sleep at high altitude. Prog Clin Biol Res 1983; 136: 75 - 85

17 Lahiri S, Meret $K$, Sherpa $M$. Dependence of high altitude sleep apnea on ventilatory sensitivity to hypoxia. Respir Physiol 1983; 52: 281 301

18 Schoene $R$. Control of ventilation in climbers to extreme altitude. J Appl Physiol 1982; 53: 886-890

19 White D, Gleeson K, Pickett $C$ et al. Altitude acclimatization: influence on periodic breathing and chemoresponsiveness during sleep. J Appl Physiol 1987; 63: $401-412$

20 Farney RJ, Walker JM, Cloward TV et al. Sleep-disordered breathing associated with long-term opioid therapy. Chest 2003; 123: $632-639$

21 Shook JE, Watkins WD, Camporesi EM. Differential roles of opioid receptors in respiration, respiratory disease, and opiate-induced respiratory depression. Am Rev Respir Dis 1990; 142: 895-909

22 Teichtahl H, Prodromidis A, Miller B et al. Sleep-disordered breathing in stable meathadone programme patients: a pilot study. Addiction 2001; 96: $395-403$

23 Committee on fetus and newborn. American Academy of pediatrics. Apnea, sudden infant death syndrome, and home monitoring. Pediatrics 2003; 111: $914-917$

24 Kahn A, Groswasser J, Franco P. Breathing during sleep in infancy. In: Loughlin G, Carroll J, Marcus C, eds. Sleep and breathing in children, Vol. 147. New York: Marcel Dekker, Inc., 2000: 405-422

25 National Institutes of Health. Consensus development conference on infantile apnea and home monitoring. Pediatrics 1987; 79: 292 - 299

26 Ramanathan R, Corwin MJ, Hunt CE et al. Cardiorespiratory events recorded on home monitors: comparison of healthy infants with those at increased risk for SIDS. JAMA 2001; 285: 2199-2207

27 Rigatto $H$. Breathing and sleep in preterm infants. In: Loughlin G, Carroll J, Marcus C, eds. Sleep and breathing in children, Vol. 147. New York: Marcel Dekker, Inc., 2000: 495-515

28 Rosen $C$. Maturation of breathing during sleep: infants through adolescents. In: Loughlin G, Carroll J, Marcus C, eds. Sleep and breathing in children, Vol. 147. New York: Marcel Dekker, Inc., 2000: 181 - 206

29 Samuels M. Apparent life-threatening events: pathogenesis and management. In: Loughlin G, Carroll J, Marcus C, eds. Sleep and breathing in children, Vol. 147. New York: Marcel Dekker, Inc., 2000: 423-442

30 Flemons WW. Clinical practice. Obstructive sleep apnea. N Engl J Med 2002; 347: 498 - 504

31 Malhotra A, White DP. Pathogenesis of obstructive sleep apnea hypopnea syndrome. In: McNicholas WT, Phillipson EA, eds. Breathing disorders during sleep. London: WB Saunders, 2002: 44-63

32 Redline S, Strohl KP, Tishler PV. The genetics of obstructive sleep apnea hypopnea syndrome. In: Pack AI, ed. Sleep apnea: Pathogenesis, diagnosis, and treatment. New York: Marcel Dekker, 2002: 235-264

33 American Academy of Sleep Medicine. Sleep-related breathing disorders in adults: recommendations for syndrome definition and measurement techniques in clinical research. The Report of an American Academy of Sleep Medicine: Task Force. Sleep 1999; 22: 667-689

34 Young T, Palta M, Dempsey J et al. The occurrence of sleep-disordered breathing among middle-aged adults. N Engl J Med 1993; 328: 1230 1235
35 Young T, Peppard PE, Gottlieb DJ. Epidemiology of obstructive sleep apnea: a population health perspective. Am J Respir Crit Care Med 2002; 165: $1217-1239$

36 American Thoracic Society. Standards and indications for cardiopulmonary sleep studies in children. Am J Respir Crit Care Med 1996. 153: $866-878$

37 Arens $R$, McDonough JM, Costarino AT et al. Magnetic resonance imaging of the upper airway structure in children with obstructive sleep apnea syndrome. Am J Respir Crit Care Med 2001; 164: 698 - 703

38 Brouillette RT, Fernbach SK, Hunt CE. Obstructive sleep apnea in infants and children. J Pediatr 1982; 100: 31 - 40

39 Guilleminault C, Partinen M, Praud JP et al. Morphometric facial changes and obstructive sleep apnea in adolescents. J Pediatr Child Health 1989; 114: $997-999$

40 Marcus CL. Pathophysiology of childhood obstructive sleep apnea: current concepts. Respir Physiol 2000; 119: 143 - 154

41 Redline S, Tishler PV, Schluchter M et al. Risk factors for sleep-disordered breathing in children. Association with obesity, race, and respiratory problems. Am J Respir Crit Care Med 1999; 159: 1527-1532

42 Plum F, Leigh R. Abnormalities of central mechanisms. In: Hornbein T, ed. Regulation of breathing. Part II. Lung biology in health and disease, Vol. 17. New York: Marcel Dekker, 1981: 989-1067

43 Sullivan C, Issa F, Berthon-Jones $M$ et al. Pathophysiology of sleep apnea. In: Saunders N, Sullivan C, eds. Sleep and breathing. Lung biology in health and diseases, Vol. 21. New York: Marcel Dekker, 1984: 299 364

44 American Thoracic Society. Idiopathic congenital central hypoventilation syndrome: diagnosis and management. Am J Respir Crit Care Med 1999; 160: $368-373$

45 Amiel J, Laudier B, Attie-Bitach $T$ et al. Polyalanine expansion and frameshift mutations of the paired-like homebox gene PHOX2B in congenital central hypoventilation syndrome. Nat Genet 2003; 33: $459-461$

46 Gozal D, Marcus C, Shoseyov D et al. Peripheral chemoreceptor function in children with the congenital central hypoventilation syndrome. J Appl Physiol 1993; 74: 379-389

47 Marcus $C$, Jansen $M$, Poulsen $M$ et al. Medical and psychosocial outcome of children with congenital central hypoventilation syndrome. J Pediatr 1991; 119: 888 - 895

48 Paton J, Swaminathan S, Sargent $C$ et al. Ventilatory response to exercise in children with congenital central hypoventilation syndrome. Am Rev Respir Dis 1993; 147: 1185- 1191

49 Paton J, Swaminathan S, Sargent C et al. Hypoxic and hypercapnic ventilatory responses in awake children with congenital central hypoventilation syndrome. Am Rev Respir Dis 1989; 140: 368 - 372

50 Bye PT, Issa F, Berthon-Jones $M$ et al. Studies of oxygenation during sleep in patients with interstitial lung disease. Am Rev Respir Dis 1984; 129: $27-32$

51 Hargrave D, Wade A, Evans $J$ et al. Nocturnal oxygen saturation and painful sickle cell crises in children. Blood 2003; 101: 846 - 848

52 Midgren B, Hansson L, Eriksson L et al. Oxygen desaturation during sleep and exercise in patients with interstitial lung disease. Thorax 1987; 42: 353 - 356

53 Needleman J, Franco M, Varlotta L et al. mechanisms of nocturnal oxyhemoglobin desaturation in children and adolescents with sickle cell disease. Pediatr Pulmonol 1999; 28: 418-422

54 Perez-Padilla $R$, West $P$, Lertzman $M$ et al. Breathing during sleep in patients with interstitial lung disease. Am Rev Respir Dis 1985; 132: $224-229$

55 Rafanan A, Golish J, Dinner D et al. Nocturnal hypoxemia is common in primary pulmonary hypertension. Chest 2001; 120: 894-899

56 Chaouat $A$, Weitzenblum E, Kessler $R$ et al. Sleep-related $\mathrm{O}_{2}$ desaturation and daytime pulmonary haemodynamics in COPD patients with mild hypoxaemia. Eur Respir J 1997; 10: 1730-1735

57 Fletcher EC, Donner CF, Midgren B et al. Survival in COPD patients with a daytime $\mathrm{PaO}_{2}$ greater than $60 \mathrm{~mm} \mathrm{Hg}$ with and without nocturnal oxyhemoglobin desaturation. Chest 1992; 101: 649-655

58 Fletcher EC, Levin DC. Cardiopulmonary hemodynamics during sleep in subjects with chronic obstructive pulmonary disease. The effect of short- and long-term oxygen. Chest 1984; 85: 6-14

59 Fletcher EC, Miller J, Divine GW et al. Nocturnal oxyhemoglobin desaturation in COPD patients with arterial oxygen tensions above $60 \mathrm{~mm} \mathrm{Hg}$. Chest 1987; 92: 604-608

60 Grant I, Heaton RK, McSweeney AJ et al. Brain dysfunction in COPD. Chest 1980; 77: $308-309$ 
61 National Institutes of Health, National Heart, Lung, and Blood Institute, World Health Organisation. Global initiative for chronic obstructive pulmonary disease. Global strategy for the diagnosis, management and prevention of chronic obstructive pulmonary disease, 2003. Available at http://www.goldcopd.com. Accessed, December 8, 2004

62 Sanders $M H$, Newman AB, Haggerty $C L$ et al. Sleep and sleep-disordered breathing in adults with predominantly mild obstructive airway disease. Am J Respir Crit Care Med 2003; 167: 7-14

63 Bye PT, Ellis ER, Issa FG et al. Respiratory failure and sleep in neuromuscular disease. Thorax 1990; 45: $241-247$
64 Hill NS, Eveloff SE, Carlisle CC et al. Efficacy of nocturnal nasal ventilation in patients with restrictive thoracic disease. Am Rev Respir Dis 1992; 145: 365 - 371

65 Hukins CA, Hillman DR. Daytime predictors of sleep hypoventilation in Duchenne muscular dystrophy. Am J Respir Crit Care Med 2000; 161: $166-170$

66 Labanowski M, Schmidt-Nowara W, Guilleminault C. Sleep and neuromuscular disease: frequency of sleep-disordered breathing in a neuromuscular disease clinic population. Neurology 1996; 47: 1173-1180

67 Lopata M, Onal E. Mass loading, sleep apnea, and the pathogenesis of obesity hypoventilation. Am Rev Respir Dis 1982; 126: 640-645

Studienaufruf

Stellenwert der Atemphysiotherapie in der Behandlung von COPD-Patienten mit Husten

Liebe Kollegen,

die Atemphysiotherapie ist eine unterstützende Säule in der Behandlung von COPD-Patienten. Wir gehen davon aus, dass COPD-Patienten von befundorientierten Behandlungstechniken und erlernten Selbsthilfemaßnahmen profitieren. Leider gibt es bisher keine methodisch einwandfreien Studien, die die Effekte der Atemphysiotherapie bei COPD-Patienten nachweisen. So sind viele Techniken derzeit „nur“ mit Evidenzgrad C belegt. Vor diesem Hintergrund plant die Arbeitsgemeinschaft-Atemtherapie im Zentralverband der Physiotherapeuten/Krankengymnasten in Zusammenarbeit mit der Deutschen Atemwegsliga e.V. die Wirksamkeit der ambulanten Atemphysiotherapie zu evaluieren.

In unserer Studie „Stellenwert der Atemphysiotherapie in der Behandlung von COPD-Patienten mit Husten“ erwarten wir für die Patienten mit Husten eine Verbesserung in verschiedenen Bereichen. Der Nachweis soll mit standardisierten Verfahren und Tests geführt werden. Durchgeführt wird eine multizentrische, randomisierte, plazebokontrollierte Studie mit drei Therapiearmen.

Insgesamt 210 Patienten werden zugeteilt auf

Gruppe „blau“: Standardtherapie und add on Physiotherapie,

Gruppe „violett“: Standardtherapie und add on „Plaze-

bo“-Physiotherapie,

Gruppe „grün“: Standardtherapie (usual care)

und in 25 bis 30 deutschen Physiotherapie-Praxen mit Schwerpunkt Atemphysiotherapie behandelt.
Charakterisierung der Patienten

Einschlusskriterien:

Diagnose COPD (Anamnese+Untersuchung: FEV 1 postbronchodilatatorisch $<50 \%$ Soll und $\Delta \mathrm{FEV}_{1}<15 \%$ nach Inhalation von $200 \mu \mathrm{g}$ Fenoterol und $80 \mu \mathrm{g}$ Ipratropiumbromid)

Subjektive Beeinträchtigung durch Husten

Ausschlusskriterien:

Atemphysiotherapie (incl. Flutter und Cornet) zur Behandlung der COPD innerhalb der letzten 6 Monate Exazerbation innerhalb der letzten 4 Wochen. Ein Einschluss ist nach Ende der Exazerbation bei einer Wartezeit von 4 Wochen selbstverständlich möglich. Änderung der COPD-Dauertherapie in den letzten vier Wochen

Zusätzliche bedeutsame Lungenkrankheit wie $z$.B. bekannte Bronchiektasie oder Lungenkarzinom Immobilisation, Herzinsuffizienz (NYHA III, IV), Demenz. Wir bitten Sie, uns bei dieser wichtigen Studie zu unterstützen.

Studienkoordinator ist Dr. Oliver Göhl, der unter 0911/9615411 (olivergoehl@nefkom.net) gerne weitere Fragen beantwortet oder Ihre Anmeldung entgegen nimmt. Pro Patient, den Sie rekrutieren, erhalten Sie eine Aufwandsentschädigung in Höhe von 50,- .

Vielen Dank für Ihre Hilfe.

Prof. Dr. Heinrich Worth

Vorsitzender der Deutschen Atemwegsliga e.V. 